\title{
Dielectric Relaxation in Cellulose and its Derivatives
}

\author{
A. Rachocki, E. Markiewicz and J. Tritt-Goc* \\ Institute of Molecular Physics, Polish Academy of Sciences \\ M. Smoluchowskiego 17, 60-179 Poznań, Poland
}

The relaxation processes in cellulose, methyl cellulose, hydroxypropyl cellulose, and hydroxypropylmethyl cellulose were studied by dielectric spectroscopy. The dielectric spectra for these polysaccharides were measured in the frequency range from $100 \mathrm{~Hz}$ to $1 \mathrm{MHz}$ and in the temperature range from 100 to $450 \mathrm{~K}$. The dielectric relaxation data for cellulose, methyl cellulose, hydroxypropylmethyl cellulose were described by Arrhenius and Eyring equations and interpreted as due to a local motion of chain segments via the glucosidic linkages, so-called $\beta$-relaxation. The same relaxation process was also determined for the hydroxypropyl cellulose polymer in the temperature range of 240-270 K. At higher temperature in hydroxypropyl cellulose another relaxation mechanism occurs due to the reorientation of the side chain built of a few hydroxypropylene groups. The activation parameters of the observed dielectric relaxation processes were determined.

PACS numbers: 61.41.+e, 77.84.Jd, 77.22.Gm

\section{Introduction}

The derivatives of cellulose, like methyl cellulose (MC), hydroxypropyl cellulose (HPC), and hydroxypropylmethyl cellulose (HPMC), are characterized by a wide field of applications. They are, for example, used in the pharmaceutical industry for production of coated and controlled release tablets and like other polysaccharides they serve as storage materials and as structural materials in plants.

In recent years the polysaccharides were the subject of many experimental and theoretical studies. Attempts have been made to correlate the properties of various polysaccharides with their chemical structure and conformation, to analyze proton NMR relaxation data, and to get structure information by analyzing 1D

*corresponding author; e-mail: jtg@ifmpan.poznan.pl 
and 2D NMR spectra [1-3]. A great number of papers have been reported on the interaction of the polysaccharides with a solvent [4-8]. The mechanical and dielectric relaxation of cellulose and its derivatives were also widely studied [9-20]. Despite the range and number of the former studies, there is still the open question of the molecular origin of the dielectric relaxation in polysaccharides.

Therefore, we undertook dielectric measurements of cellulose, MC, HPC, and HPMC as a function of temperature and frequency. In the case of dielectric relaxation the molecule has to possess a permanent dipole moment in order to undergo relaxation. However, the permanent dipolar moments of the repeated unit of the polymer and the attached side groups will be the source of the dielectric relaxation only when they fluctuate in time. The molecular motions cause the fluctuation of the dipolar moments. Therefore, the main goal of our study is to determine the molecular origin of the dielectric relaxation in cellulose, MC, HPC, and HPMC and to study the influence of the side groups on the relaxation.

\section{Experimental}

\subsection{Materials}

The samples of cellulose, methyl cellulose, hydroxypropyl cellulose, and hydroxypropylmethyl cellulose were purchased from Aldrich Chemical (Poznań, Poland). The number-averaged molecular mass $M_{n}$ is equal to 74000,17000 , 10000, and 12000 for cellulose, MC, HPC, and HPMC, respectively. The materials were used as purchased.

The cellulose and its derivatives are characterized by the same monomer residues and the same type of linkage that connects residues in the polymer chain. The building blocs are the glucose units (Fig. 1) which are linked in the polymer chain via oxygen atoms forming a so-called glucosidic linkage [14]. Each of the glucose units carries the following side groups: two $(-\mathrm{OR})$ and one $\left(-\mathrm{CH}_{2} \mathrm{OR}\right)$ groups. For cellulose the $\mathrm{R}$ symbol stands for the hydrogen atoms, thus we have two hydroxyl groups $(-\mathrm{OH})$ and one methylol group $\left(-\mathrm{CH}_{2} \mathrm{OH}\right)$ attached to the

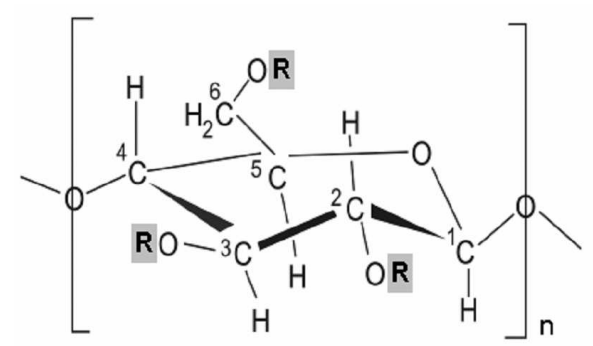

Fig. 1. Schematic representation of the glucose - the constitutive unit of the cellulose $[\mathrm{R}=\mathrm{H}], \mathrm{MC}\left[\mathrm{R}=\mathrm{H}\right.$ or $\left.\mathrm{CH}_{3}\right], \mathrm{HPC}\left[\mathrm{R}=\mathrm{H}\right.$ or $\left.k \cdot\left(\mathrm{CH}_{2}-\mathrm{CH}(\mathrm{OH})-\mathrm{CH}_{3}\right)\right]$, and HPMC $\left[\mathrm{R}=\mathrm{H}\right.$ or $\mathrm{CH}_{3}$ or $\left.\mathrm{CH}_{2}-\mathrm{CH}(\mathrm{OH})-\mathrm{CH}_{3}\right]$. 
glucose ring. For MC, R can be replaced by hydrogen atoms or methyl groups. In HPC $\mathrm{R}=\mathrm{H}$ or $k \times$ hydroxypropylene groups, where $k$ means the number of attached groups. A single hydroxypropylene groups, $\mathrm{H}$, or $\mathrm{CH}_{3}$ replace $\mathrm{R}$ in the case of HPMC. In the studied polymers the substitution of the functional groups is unknown. Therefore, we have to assume that the side groups are distributed statistically among the polymer chains.

\subsection{Dielectric measurements}

The dielectric cylinder samples with a diameter of $8 \mathrm{~mm}$ and a thickness of $0.7 \mathrm{~mm}$ were obtained by pressing the powder materials of cellulose, MC, HPC, and HPMC using a hydraulic press with $1000 \mathrm{MPa}$ at room temperature. The plates with gold cylinder capacitors were evaporated by a BAL-TEC SCD 050 sputter coater at room temperature. Dielectric dispersion and absorption was studied in the frequency range from $100 \mathrm{~Hz}$ to $1 \mathrm{MHz}$ using a computer controlled HP-4284A precision meter. The measurements were performed during heating from 100 to $450 \mathrm{~K}$ at a rate of $1 \mathrm{~K} / \mathrm{min}$.

\section{Results and discussion}

\subsection{Dielectric spectra and Arrhenius plot of relaxation times}

The dielectric spectra of the measured samples can be presented in the form of the real part of the complex dielectric function $\varepsilon^{\prime}$ and/or imaginary part $\varepsilon^{\prime \prime}$ versus the frequencies and/or the temperature. The experimental data of $\varepsilon^{\prime \prime}$ as a function of frequency and temperature are shown in Fig. 2, for cellulose, MC, HPC, and HPMC, respectively. The relaxation processes observed in the lower temperature range are the most interesting, because they yield information about the molecular dynamics of the polymer systems. In Fig. 3 the activation plot (Arrhenius plot of $\tau$ versus $1 / T$ ) of the temperature dependence on the dielectric relaxation $\tau$ for cellulose, MC, HPC, and HPMC is shown. The dielectric relaxation data are plotted on a logarithmic scale.

In the higher temperature range we can observe in Fig. 2 the $\beta_{\text {wet }}$ relaxation, which is due to water content in studied polysaccharides, whereas in the highest temperature range (and lower frequency range) we can also see the conductivity effect, which is especially visible for HPC and HPMC (Fig. 2c and d).

\subsection{Discussion and interpretation on dielectric data}

Cellulose and its derivatives have been the subject of extensive study by dielectric methods [9-20]. The main goal of these studies was to assign the single relaxation processes found in the experiment to the corresponding molecular orientational processes of the polysaccharides. In dielectric relaxation studies of polymers, two processes are usually distinguished: the primary relaxation process 


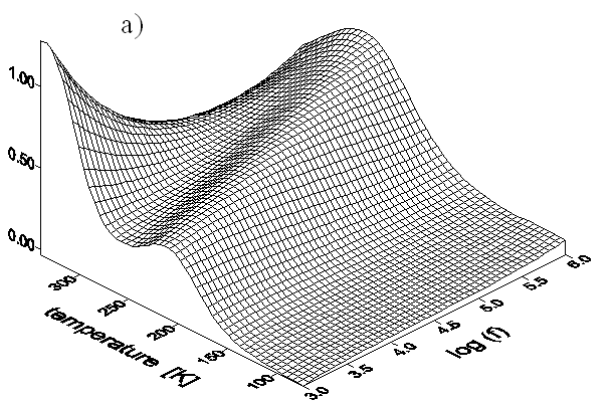

b)
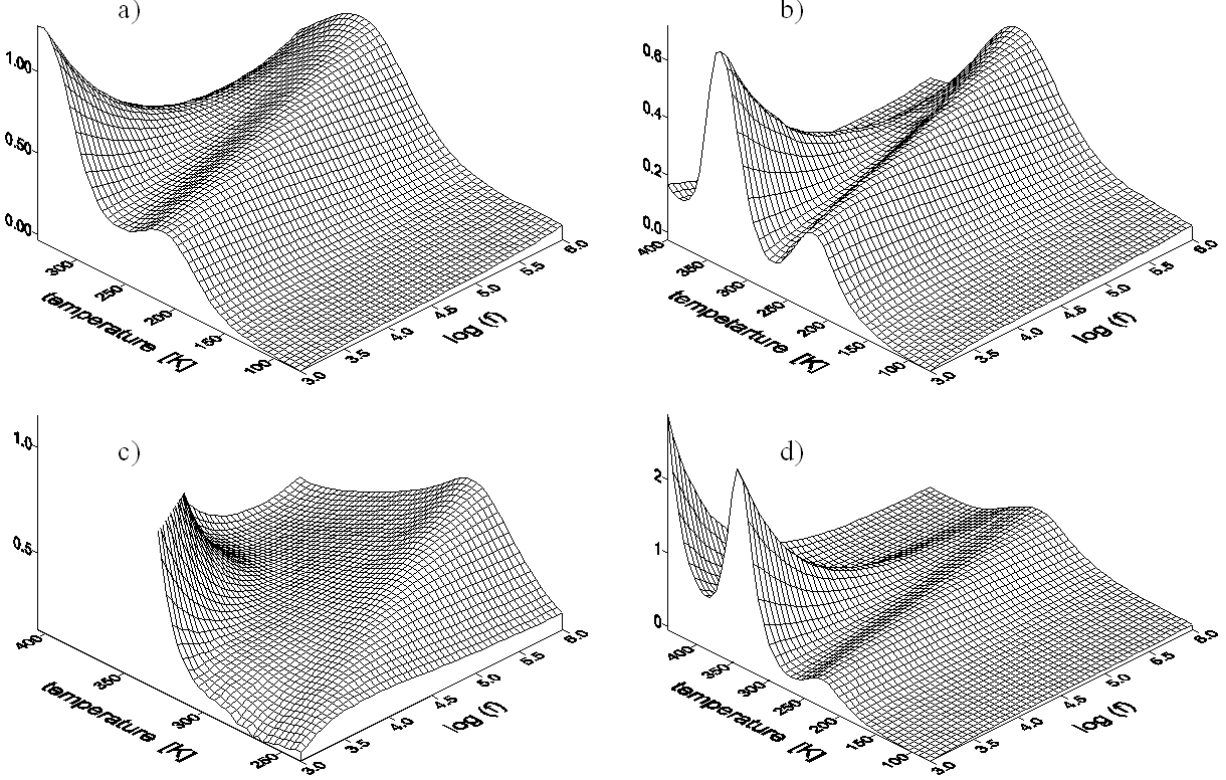

Fig. 2. The imaginary part of dielectric permittivity $\varepsilon^{\prime \prime}$ vs. temperature and frequency for (a) cellulose (b) MC, (c) HPC, and (d) HPMC.

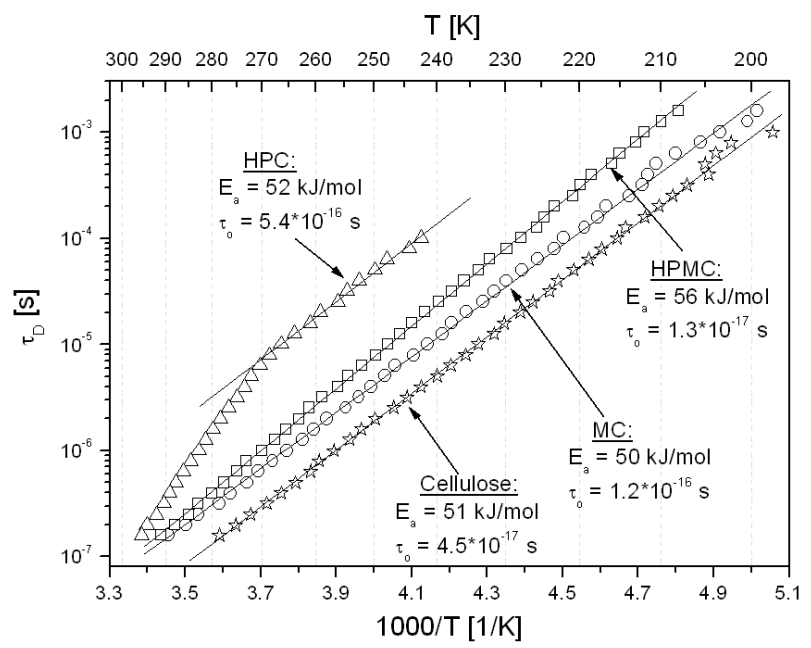

Fig. 3. The Arrhenius plots of the temperature dependence on the dielectric relaxation times for cellulose, MC, HPC, and HPMC.

and several secondary relaxations [21]. The primary or $\alpha$-relaxation is related to the glass transition and is observed at the temperature above the glass transition temperature whereas the secondary relaxation processes are usually observed below the glass temperature. Among them the most important are: $\beta$-relaxation, 
$\gamma$-relaxation, and $\sigma$-relaxation. The $\beta$-relaxation process in polysaccharides is related to segmental motion of the polysaccharide chain via the glucosidic bond and therefore corresponds to the local chain motion. The $\gamma$-relaxation is related to the motion of the side groups attached to the glucosic unit of the polysaccharides, whereas the $\sigma$-relaxation process, observed at the high temperatures, involves proton migration or ion hopping in amorphous polymers.

For many years the secondary relaxations observed in polysaccharides were discussed in papers in a controversial way. The most common was to interpret the low temperature relaxation data in cellulose or its derivatives as $\gamma$-relaxation, thus connected with the side group motion [9-14]. Some other authors interpreted this relaxation as the local chain motion ( $\beta$-relaxation) $[15,16]$, while others tried to explain the experimental results by relating the relaxation to the reorientation of bound water molecules in the polymers [17]. The paper which put in order the interpretation of the dielectric relaxation in polysaccharides in the low temperature range, are the ones by Meissner et al. [18, 19]. They studied the glucose monomer, dimmer cellbiose, pentamer cellopentose, and cellulose polymer. On the basis of the dielectric spectra of these compounds they concluded that the main relaxation process found in all pure polysaccharides in the temperature range from $138 \mathrm{~K}$ to $295 \mathrm{~K}$ is the $\beta$-relaxation, whereas the $\gamma$-relaxation is not separately visible in the frequency window between $1 \mathrm{MHz}$ and $3 \mathrm{MHz}$. In this frequency range the local chain motion relaxation overlaps the side group mobility. The contribution to the molecular origin of the dielectric relaxation in polysaccharides at the high temperature is discussed by Einfeldt et al. [20].

The description of the relaxation processes is given by the model function of Havriliak and Negami [22, 23]

$$
\Delta \varepsilon^{*}=\Delta \varepsilon_{i} /\left[1+\left(\mathrm{i} \omega \tau_{1}\right)^{a i}\right]^{b i},
$$

where $a$ is the parameter which characterises the width and $b$ - the asymmetry of the relaxation processes. If $a=b=1$ then the relaxation process is simply a Debye process. In such a case of a symmetrical relaxation process the relaxation time $\tau_{i}(T)$ represents the central position of the process on the time scale and is equal to the inversion of the maximum frequency: $\tau_{i}=1 /\left(2 \pi f_{\max }\right)$. The temperature dependence on the relaxation time $\tau(T)$ follows the Arrhenius behaviour:

$$
\begin{aligned}
& \tau(T)=\tau_{0} \exp \left(E_{\mathrm{a}} / R T\right), \\
& \tau(T)=\tau_{\mathrm{D}} \exp \left(-\Delta S_{\mathrm{a}} / R\right) \exp \left(\Delta H_{\mathrm{a}} / R T\right),
\end{aligned}
$$

where $E_{\mathrm{a}}$ denotes the activation energy, $\Delta H_{\mathrm{a}}$ - the excess enthalpy, $\Delta S_{\mathrm{a}}-$ the excess entropy, $\tau_{\mathrm{D}}=h / k T_{0}=1.76 \times 10^{-13} \mathrm{~s}, R$ - the universal gas constant, and $T_{0}=273.15 \mathrm{~K}$. Equation (2b) derived by Eyring in [24] correlates the deviation of the pre-exponential factor $\tau_{0}$ from the Debye $\tau_{\mathrm{D}}$-value to the excess entropy due to the reorientation in the relaxation process. High entropy values or a smaller value of $\tau_{0}$ than $\tau_{\mathrm{D}}$ can be interpreted to arise from co-operativity of the orientational motion of the neighbouring side chain. 
Equation (2a) was used to fit the experimental data of the temperature dependence of the dielectric relaxation $\tau$ presented in Fig. 3 for cellulose, MC, HPC, and HPMC. The solid lines represent the best fits of Eq. (2a) with the parameters shown in the Table and in Fig. 3. The experimental points were fitted quite well with one line in the whole temperature range for cellulose, MC, and HPMC. Therefore we conclude that the single mechanism is responsible for the dielectric relaxation observed in these polymers. In contrary, two fitting lines were needed to describe the experimental results obtained for HPC. Thus we can assume that two different relaxation processes occur in this polymer: one in the temperature range from 244 to $270 \mathrm{~K}$ and the other one in the range of 270 to $298 \mathrm{~K}$.

TABLE

Parameters estimates derived from the fitting of Eq. (2a) and (2b) to the experimental data presented in Fig. 3.

\begin{tabular}{l|c|c|c|c}
\hline \hline & \multicolumn{2}{|c|}{ Fit of Eq. (2a) } & \multicolumn{2}{c}{ Fit of Eq. (2b) } \\
\cline { 2 - 5 } & $\begin{array}{c}E_{\mathrm{a}} \\
{[\mathrm{kJ} / \mathrm{mol}]}\end{array}$ & $\begin{array}{c}\tau_{0} \\
{[\mathrm{~s}]}\end{array}$ & $\begin{array}{c}\Delta H \\
{[\mathrm{~kJ} / \mathrm{mol}]}\end{array}$ & $\begin{array}{c}\Delta S \\
{[\mathrm{~kJ} / \mathrm{K} \mathrm{mol}]}\end{array}$ \\
\hline Cellulose $_{(\beta)}$ & 51 & $4.5 \times 10^{-17}$ & 49 & 0.06 \\
$\mathrm{MC}_{(\beta)}$ & 50 & $1.2 \times 10^{-16}$ & 48 & 0.05 \\
$\mathrm{HPMC}_{(\beta)}$ & 56 & $1.3 \times 10^{-17}$ & 54 & 0.07 \\
$\mathrm{HPC}_{(\beta)}$ & 52 & $5.4 \times 10^{-16}$ & 50 & 0.04 \\
$\operatorname{HPC}_{(\beta+\gamma)}$ & - & - & 96 & 0.2
\end{tabular}

The values of the activation energies and $\tau_{0}$ are similar for cellulose, MC, HPMC, and HPC, but in the last case only in the temperature range from $244-270 \mathrm{~K}$, pointing to the same relaxation process. The process was interpreted as $\beta$-relaxation related to the local chain motion of the polymers.

The $\tau_{0}$ values obtained from the fitting procedure are of the order of $10^{-16} \mathrm{~s}$ (for MC and HPC) and $10^{-17} \mathrm{~s}$ (for cellulose and HPMC). They are much shorter than the Debye relaxation time which indicates that the motions associated with the measured relaxation processes are the cooperative local chain segmental motions of the polysaccharides. In cellulose for example such cooperative motion involves more than two, but less than 5 repeating units [18]. It is rather obvious that the main chain motion is also coupled with the reorientation of the side groups. A smaller value of $\tau_{0}$ means a higher cooperativity of the motion. HPMC differs from MC only by the large hydroxypropylene functional group. There is a higher possibility for this group to be involved in the inter-chains hydrogen bondings due to the higher dimension (volume and mass) when compared to methoxy and methylenemethoxy groups, present in the MC polymer. Therefore, the local chain motion in HPMC can involve not only the nearest repeating unit of the same chain, but also through the side groups, those from neighbouring chain. 
Consequently, the lower value of the pre-exponential factor $\tau_{0}$ was obtained for hydroxypropylmethyl cellulose when compared to methyl cellulose. As can be see in Fig. 3, HPMC is characterised by the highest value of the activation energy, that is $56 \mathrm{~kJ} / \mathrm{mol}$ when compared to $52 \mathrm{~kJ} / \mathrm{mol}, 51 \mathrm{~kJ} / \mathrm{mol}$, and $50 \mathrm{~kJ} / \mathrm{mol}$ for HPC, cellulose, and MC, respectively. It seems to be the consequence of the large hydroxypropylene functional groups bound to the main building blocs of the HPMC polymer. Such groups, having large dimensions, make the chains of HPMC polymer more rigid, thus the oscillations of the glucose rings become more restricted and consequently a higher value of activation energy is observed in HPMC when compared to other studied polymers.

In order to analyze the relaxation results for HPC in the high temperature range of 270-298 K, we have assumed an additional relaxation process. This second relaxation process observed only in HPC is due to the motion of the long side group bound to the glucose unit. In HPC, like in other polysaccharides, we can distinguish the main chain built of the glucose unit, but also the side chain which is built of $k$ hydroxypropylene groups. If such a side chain is long enough, the dipolar moment of it can be the source of the dielectric relaxation along with the relaxation of the main chain segment. Because this relaxation is related to the side group motion, we therefore called it the $\gamma$-relaxation. On the other hand it is known from the literature that the $\gamma$-relaxation usually occurs at low temperature, followed by local chain motion - $\beta$-relaxation, at higher temperature [9-14]. In cellulose, MC, HPMC (in the range of 240-298 K) and also in HPC (in the range of 240-298 K) we see only the $\beta$-process, because the local chain motion relaxation overlaps the mobility of the small side groups. When the side groups are large enough they can influence the dielectric relaxation and can be the source of the separate relaxation process. However the motion of the large side group is thermally activated only at a high temperature. That is the reason why the $\gamma$-relaxation process connected to the reorientation of the side chain of $k$ hydroxypropylene groups in HPC started at higher temperature than the $\beta$-process due to the local motion of chain segments via the glucosidic linkages. Therefore, we conclude that in the temperature range from 270 to $298 \mathrm{~K}$ the combined effect of $\beta$ - and $\gamma$-relaxation is seen.

The experimental data showing the dependence of the dielectric relaxation time as a function of temperature in Fig. 3 were also fitted to Eq. (2b). The calculated entropy and enthalpy contributions $(\Delta S$ and $\Delta H)$ to the free activation energy of thermo activated motions are given in the Table. For all studied polymers $\Delta S \neq 0$. This indicates that the conformational changes of the main chain of the polymer include also the motion of the side polymers groups and, as a consequence, the correlated motion is observed. The highest value of the enthalpy $\Delta H=$ $0.2 \mathrm{~kJ} / \mathrm{Kmol}$ occurs for HPMC. Therefore, we can conclude that in this polymer the motion is more correlated than in other polysaccharides.

Our experimental results for cellulose and its derivatives are in a good correspondence with the literature results found for the dielectric activation energy 
$E_{\text {a }}$ obtained for the $\beta$-relaxation process in other polysaccharides [19]. It is known that the activation energy of the local backbone motion of the polymers in solid state depends on the chain lengths measured as a degree of polymerisation (DP). This correlation has a maximum at around $\mathrm{DP}=7$ to 12 . For studied polymers DP falls in this range, which explains the rather high value of the measured activation energy. The other factor which strongly influences the value of the activation energy is the content of water. A higher amount of water in the polymer increases the value of the activation energy.

\section{Conclusion}

In the temperature range from $240 \mathrm{~K}$ to $298 \mathrm{~K}$ for cellulose, MC, and HPMC, and in the range of $240 \mathrm{~K}$ to $270 \mathrm{~K}$ for HPC the segmental motion of the main chains via the glucosidic bond contributes to the observed dielectric relaxation. In agreement with the literature devoted to polysaccharides [9-20] this relaxation is called the $\beta$-relaxation process. For HPC in addition to the main chain motion also the influence of the reorientation of the side chain of $k$ hydroxypropylene groups, in the temperature region of $240 \mathrm{~K}$ to $270 \mathrm{~K}$, influences the relaxation in such a degree that it is apparent as the second relaxation process, the so-called $\gamma$-relaxation process. The possibilities of such an observation were predicted in the literature [19].

\section{Acknowledgments}

The work was supported by the Centre of Excellence for Magnetic and Molecular Materials for Future Electronics within the European Commission Contract No. G5MA-CT-2002-04049.

\section{References}

[1] B.R. Faber, P. Erbel, J.F.G. Vliegennthart, J. Bitech, J. Biotech. 77, 115 (2000).

[2] M. Geppi, R.K. Harris, A.M. Kenwright, B.J. Say, Solid State NMR 12, 15 (1998).

[3] M. Tylianakis, A. Spyros, P. Dais, F.R. Taravel, A. Perico, Carbohydr. Res. 315, 16 (1999).

[4] R.W. Bowtell, J.C. Sharp, A. Peters, P. Mansfield, A.R. Rajabi-Siahboomi, M.C. Davies, C.D. Melia, Magn. Reson. Imaging 12, 361 (1994).

[5] A.R. Rajabi-Siahboomi, R.W. Bowtell, P. Mansfield, A. Henderson, M.C. Davies, C.D. Melia, J. Control. Rel. 3, 121 (1994).

[6] C.A. Fyfe, A.I. Blazek, Macromolecules 37, 6230 (1997).

[7] P. Colombo, R. Bettini, N.A. Peppas, J. Control. Rel. 61, 83 (1999).

[8] J. Tritt-Goc, N. Piślewski, J. Control. Rel. 80, 79 (2002).

[9] S.A. Bradley, S.H. Carr, J. Polym. Sci.-Polym. Phys. B 14, 111 (1997). 
[10] H. Montes, K. Mazeau, J.Y. Cavaille, Macromolecules 30, 6977 (1997).

[11] H. Montes, K. Mazeau, J.Y. Cavaille, J. Non-Cryst. Solids 235, 416 (1998).

[12] H. Montes, J.Y. Cavaille, Polymer 40, 2649 (1999).

[13] D.J. Crofton, R.A. Pethric, Polymer 23, 1609 (1982).

[14] D.J. Crofton, R.A. Pethric, Polymer 22, 1048 (1981).

[15] M. Scandola, G. Ceccorulli, M. Pizzoli, Int. J. Biol. Macromol. 13, 254 (1991).

[16] M.F. Butler, R.E. Cameron, Polymer 41, 2249 (2000).

[17] V.J. McBrierty, C.M. Keely, F.M. Coyle, X. Huan, J.K. Vij, Farady Discuss. 103, 255 (1996).

[18] D. Meissner, J. Einfeldt, K. Kwasniewski, J. Non-Cryst. Solids 275, 199 (2000).

[19] J. Einfeldt, D. Meissner, K. Kwasniewski, Prog. Polym. Sci. 26, 1419 (2001).

[20] J. Einfeldt, D. Meissner, K. Kwasniewski, J. Non-Cryst. Solids 320, 40 (2003).

[21] F. Kremer, A. Sconhals, Brodband Dielectric Spectroscopy, Springer-Verlag, Berlin 2003.

[22] S. Havriliak, S. Negami, J. Brit, J. Appl. Phys. 2, 1301 (1969).

[23] S. Jr. Havriliak, S. Havriliak, J. Polym. 37, 4107 (1996).

[24] S. Glasstone, K.J. Laidler, H. Eyring, The Theory of Rate Processes, McGraw Hill, New York 1941. 